\title{
HIV/AIDS: evolução e depressão em pessoas soropositivas: uma revisão narrativa
}

\author{
HIV/AIDS: evolution and depression in HIV-positive people: a narrative review
}

VIH/SIDA: evolución y depresión em personas soropositivas: uma revisión narrativa José Doriberto Freitas $^{1 *}$, Regina Heloisa Mattei de Oliveira Maciel ${ }^{1}$.

\begin{abstract}
RESUMO
Objetivo: Verificar a evolução da depressão em pessoas que vivem com o vírus da Imunodeficiência Humana/Síndrome da Imunodeficiência adquirida (HIV/AIDS) na atualidade. Revisão bibliográfica: Durante a seleção dos artigos, foi observado que a maioria das publicações sobre a temática Depressão e HIV/AIDS enfatiza os aspectos psicopatológicos e/ou epidemiológicos da depressão e que a pessoa portadora da síndrome convive com a depressão que causa sofrimento e incapacidade perante a vida. Pode-se perceber uma estreita relação entre depressão e HIV/AIDS no que se refere aos seus impactos negativos na saúde e qualidade de vida dessa população. Há variedade de sintomas apresentados pelos indivíduos: alteração do humor e do afeto, tristeza profunda, perda de prazer, aumento e diminuição do apetite e alterações da fisiologia do sono. Considerações finais: A luta contra a depressão em pessoas vivendo com HIV/AIDS exige esforços e apoio familiar e social, bem como um posicionamento governamental e incentivo à comunidade técnico-científica para proporcionar uma melhor qualidade de vida para este segmento tão estigmatizado e marginalizado.
\end{abstract}

Palavras-chave: Depressão, AIDS, HIV, Perfil epidemiológico, Evolução.

\begin{abstract}
Objective: The aim was to verify today's evolution of depression in people living with the Human Immunodeficiency Virus/Acquired Immunodeficiency Syndrome (HIV/AIDS). Bibliographic review: During the selection of articles, it was observed that most publications on the theme Depression and HIV/AIDS emphasizes the psychopathological and/or epidemiological aspects of depression and that the person with the syndrome lives with depression, that causes suffering and disability in life. There is a close relationship between depression and HIV/AIDS about its negative impacts on health and quality of life of this population. A variety of symptoms are presented in those individuals: changes in mood and affection, deep sadness, loss of pleasure, increase and decrease in appetite and changes in sleep physiology. Final considerations: The fight against depression in people living with HIV/AIDS requires efforts, family, and social support, as well as governmental policies and encouragement to the technical-scientific community to provide a better quality of life for this stigmatized and marginalized segment.
\end{abstract}

Key words: Depression, HIV, AIDS, Epidemiological profile, Evolution.

\section{RESUMEN}

Objetivo: Verificar la evolución de la depresión em personas que viven con el Virus de Inmunodeficiencia Humana/Síndrome de Inmunodeficiencia Adquirida (VIH/SIDA) em la actualidad. Revisión bibliográfica: Durante la selección de artículos, se observó que la mayoría de las publicaciones sobre el tema Depresión y $\mathrm{VIH} / \mathrm{SIDA}$ enfatizan los aspectos psicopatológicos y/o epidemiológicos de la depresión y que la persona con

${ }^{1}$ Universidade de Fortaleza (UNIFOR), Fortaleza - CE. *E-mail: doribertofreitas@gmail.com 
el síndrome vive con depresión que le causa sufrimiento y discapacidad ante la vida. Se puede percibir una variedad de síntomas que presentan los individuos: cambios en el estado de ánimo y el afecto, tristeza profunda, perdida de placer, aumento y disminución del apetito y cambios en la fisiología del sueño. Consideraciones finales: La lucha contra la depresión en personas que viven con VIH/SIDA requiere esfuerzos y apoyo familiar y social, así como una postura gubernamental y estímulo a la Comunidad técnicocientífica para brindar una mejor calidad de vida a este segmento tan estigmatizado y marginalizado.

Palabras clave: Depresión, VIH, SIDA, Perfil epidemiológico, Evolución.

\section{INTRODUÇÃO}

Na década de 1980, os Centros de Controle e Prevenção de Doenças dos Estados Unidos da América, identificaram uma nova e misteriosa síndrome com base em casos de pneumonia atípica e de um câncer raro do tipo Sarcoma de Kaposi em homens jovens, previamente saudáveis, em sua maioria homossexuais masculinos, nas cidades de Los Angeles, São Francisco e Nova York (JARDIM E, 2019).

Após inúmeros avanços e retrocessos, erros e acertos por parte de epidemiologistas e infectologistas, os pesquisadores chegaram à definição e caracterização da Síndrome da Imunodeficiência Adquirida (cuja sigla é SIDA ou AIDS, esta última em língua inglesa, adotada no Brasil) que tem como agente etiológico o Vírus da Imunodeficiência Humana (HIV). O vírus infecta e mata as células T CD4 e macrófagos, ambos críticos para a resposta imune efetiva. O HIV é transmitido através da exposição da mucosa oral, vaginal ou retal durante as práticas sexuais ou durante a amamentação, assim como pela inoculação intravascular, por transfusão de sangue ou hemoderivados e utilização de equipamentos infectados, compartilhamento de seringas durante a injeção de drogas ou através da transmissão vertical da circulação materno fetal (CAMPIOTTO LG, et al., 2013).

Existem dois grupos de portadores da síndrome. Pacientes com HIV, portadores do vírus, mas assintomáticos, que ainda não desenvolveram a doença, e pacientes com AIDS, sintomáticos, que já manifestaram alguma doença oportunista em decorrência da ação do HIV. Em termos de qualidade de vida, bem como vivências intrapsíquicas, parece haver diferenças entre os dois grupos como conviver apenas com o vírus, no caso dos assintomáticos, e ter desenvolvido doenças clínicas na vivência dos sintomáticos (BRIAN KB, et al., 1998).

A alta prevalência de morbidade e mortalidade desde os primeiros casos de HIV e a identificação do vírus, a AIDS transformou-se em um grande problema de saúde pública e, desde então, diversos desafios vêm sendo colocados à humanidade por seu caráter pandêmico com rápida disseminação e agravamento. Ainda de acordo com os autores o perfil epidemiológico e as tendências iniciais de infecção e transmissão por drogadictos, homossexuais, profissionais do sexo, foram se modificando ao longo dos anos. Inicialmente, acreditava-se que a doença atingia apenas determinados grupos de "risco" como prostitutas, homens homossexuais e usuários de drogas. Pouco tempo depois, a doença foi identificada em outros segmentos da população como bissexuais, hemofílicos, parceiros de heterossexuais portadores do HIV e receptores de sangue de hemoderivados (CAMARGO LA, et al., 2014).

Atualmente a população que convive com a AIDS é caracterizada também por pessoas que residem no interior das pequenas cidades, heterossexuais, pessoas idosas, com baixa escolarização, baixo nível socioeconômico, mulheres e adolescentes que estão iniciando sua vida sexual sem nenhuma orientação (PEDROSA SC, et al., 2016). De acordo com Camargo LA, et al. (2014) a infecção entre heterossexuais é, na atualidade, a subcategoria de exposição sexual com maior número de notificações.

De acordo com o relatório UNAIDS (2020), no seu resumo global de epidemiologia, existem, atualmente, cerca de 38 milhões de pessoas vivendo com HIVIAIDS, dos quais 17 milhões são homens, 19,2 milhões são mulheres e 1,8 milhão são crianças com menos de 15 anos de idade. Quanto às pessoas recém infectadas pelo HIV, estas somam 1,7 milhões, totalizando 1,5 milhão de adultos e 150.000 crianças menores de 15 anos. 
No que se refere às mortes relacionadas à AIDS, estas totalizam 690.000 mil, sendo 600.000 mil de adultos e 95.000 crianças com menos de 15 anos. Apesar da disponibilidade de tratamentos eficazes, $62 \%$ das novas infecções por HIV em adultos estão entre populações chave e seu parceiro sexual; meninas adolescentes e mulheres jovens contabilizam 1 em 4 infecções na África Subsariana. Além disso, constatou-se que, em 25 países, mais de $50 \%$ de adultos têm atitudes discriminatória para com as pessoas vivendo com HIV (UNAIDS, 2020).

No Brasil, desde o início da identificação da epidemia em 1980, até dezembro de 2019, foram identificados 349.784 óbitos (MS, 2020). Segundo dados epidemiológicos apurados pela secretaria de vigilância em saúde no intervalo de 2007 a 2018 , constatou-se uma soma de 169.932 novos casos, sendo $(68,6 \%)$ em homens e $77.82(31,4 \%)$ correspondentes a mulheres (DIAS WB, et al., 2020).

De acordo com o Manual Diagnóstico e Estatístico de Transtorno Mental (DSM-V), o transtorno depressivo é apontado como uma psicopatologia mundial, e sua característica essencial é a oscilação do humor ou do aspecto afetivo, denominado de "transtorno do humor". É uma psicopatologia que atinge o ser humano na sua constituição corporal, mental e espiritual, bem como prejudica os vínculos interpessoais (APA 2014; OMS 2017). O transtorno depressivo é classificado em níveis leve, moderado e grave. Dados da World Health Organization (WHO, 2017) apontam que atualmente existem no mundo mais de 300 milhões de pessoas com depressão.

A depressão é apontada como a principal causa de mortes por suicídio, que totalizam cerca de 800 mil anualmente. Cerca de $15 \%$ das pessoas com depressão grave cometem suicídio (OMS, 2017). De acordo com Oliveira LS, et al. (2020), o transtorno depressivo é um estado que faz com que os indivíduos se sintam tristes continuamente e percam a motivação por tarefas que anteriormente davam prazer. Os sintomas apresentados podem consistir em afastamento social, falta de esperança e sentir-se sem utilidade, insônia, falta de apetite e perda de peso, esgotamento, falta de concentração, pensamentos e ideias suicidas.

A depressão tornou-se uma problemática de grande evidencia e parece estar relacionada a uma reação ao mundo contemporâneo. O Relatório Mundial de Saúde (ROCHA RFC, 2017) mostra que a urbanização, o envelhecimento e as modificações globalizadas do estilo de vida, fazem com que doenças crônicas e não transmissíveis, incluindo a depressão, sejam motivos cada vez mais importantes de morbidades e de mortalidade. Suas causas perpassam o meio psicossocial, embora sejam reconhecidas certa predisposição genética. As causas psicossociais contemplam fatos que dizem respeito ao meio ambiente, como o estresse da vida diária (KAPLAN HI e SADOCK B, 2016).

Whitbourne SK e Halgin RP (2015) citam que a prevalência da depressão ao longo da vida nos Estados Unidos é de $16,6 \%$ da população adulta. A cada ano, 6,7\% da população adulta recebe um diagnóstico de depressão, sendo $30,4 \%$ desses casos ( $2 \%$ da população adulta) classificados como graves. As mulheres são $70 \%$ mais propensas que os homens a vivenciarem a depressão em algum momento da vida. $\mathrm{A}$ idade média de início da doença é de 32 para as mulheres. No Brasil, a Pesquisa Nacional de Amostra de Domicílios (PNAD), realizada em 2008, estimou a prevalência da depressão na população geral em 4,1\% (IBGE, 2010).

Existe uma estreita relação entre depressão e HIVIAIDS impactando negativamente a saúde e qualidade de pessoas soropositivas. Nesse sentido o presente estudo objetiva verificar a evolução da depressão em pessoas que vivem com o vírus da Imunodeficiência Humana/Síndrome da Imunodeficiência adquirida (HIVIAIDS) na atualidade, por meio de uma revisão de literatura.

\section{REVISÃO BIBLIOGRÁFICA}

A infecção pelo HIV e as desordens psiquiátricas apresentam estreita relação e têm recebido atenção especial na última década, considerando o seu impacto na vida pessoal, sexual, social e ocupacional das pessoas vivendo com o HIVIAIDS (GIOVELLI GRM, 2009). A depressão, quando vivenciada por pessoas em condição de doença crônica, pode causar incapacidade, afetando a evolução da doença, interferindo na recuperação e, ainda, é considerada risco potencial para o aumento da morbidade e mortalidade (APA, 2014). 
As repercussões psíquicas da infecção pelo HIV, como doença crônica severa e incurável, colocam o infectado à iminência da morte (BALDIM L, et al., 2016). Desse modo, pacientes soropositivos portadores do vírus e vivendo com a doença, têm se tornado objeto de interesse por parte dos profissionais de saúde mental. Esse interesse se dá pela dimensão do impacto psicológico consequente ao diagnóstico como tristeza, sentimentos de negação, hostilidade, culpa, revolta, ansiedade, angústias, transtorno do sono e vulnerabilidade social e emocional (GIOVELLI GRM, 2009).

Além da depressão, em pessoas vivendo com HIVIAIDS, observa-se que eventos de vida estressantes como desemprego, preconceito, estigma, condições socioeconômicas e fatores socioculturais desfavoráveis e conflitos familiares estão associados ao aumento da progressão da infecção pelo HIVIAIDS que, por sua vez, aumentam de três a cinco vezes o risco para o desenvolvimento de depressão (REIS RK, et al., 2011). De acordo com Reis RK, et al. (2011), a infecção pelo HIVIAIDS compromete a qualidade de vida de seu portador, devido à estreita relação entre depressão e qualidade de vida.

Camargo LA, et al. (2014) realizaram uma pesquisa em um grande hospital de São Paulo, que tinha como objetivo avaliar a prevalência de depressão e outros transtornos depressivos em 120 mulheres infectadas pelo HIV, assim como comparar a prevalência entre pacientes assintomáticas e sintomáticas para HIV. Constataram 25,8\% mulheres apresentavam depressão, sendo 13,3\% pacientes assintomáticas e 38,3\% sintomáticas. É importante lembrar que pelo menos $48 \%$ das participantes já haviam experimentado, no mínimo, um episódio de depressão, antes ou depois do diagnóstico de HIV (CAMARGO LA, et al., 2014).

Brian KB, et al. (1998) realizaram um estudo que visou investigar a prevalência e preditores de ideação e tentativas passadas de suicídio em homens homo e bissexuais, soropositivos $(n=164)$ e soronegativos $(n=$ 65) para HIV. Os pacientes sintomáticos para HIV $(n=85)$ apresentaram níveis mais altos de ideação que os assintomáticos $(n=79)$ e os soronegativos. Foram encontrados altos níveis de tentativas de suicídio tanto em soronegativos $(29 \%)$ quanto soropositivos $(21 \%)$. Dentre os fatores preditivos associados destacaram-se histórico de transtorno psiquiátrico (particularmente depressão), uso de drogas e casos de suicídio na família.

Um ponto importante no que diz respeito à relação entre depressão e HIVIAIDS é a falta de instrumentos específicos para identificar o problema. Porém numa pesquisa realizada por Coutinho MFC, et al. (2018) que tinha como objetivo identificar se a depressão interfere na adesão ao tratamento antirretroviral concluiu-se que a relação entre depressão e não adesão não se constatou, apesar de ter se verificado a prevalência de depressão de $22,24 \%$ da amostra.

Silva IBN, et al. (2020) realizaram um estudo com objetivo de analisar a esperança de vida e a depressão em pessoas vivendo com HIVIAIDS. Concluíram que a fé e a esperança são valores importantes, contribuindo para a habilidade em lidar com situações difíceis e na manutenção da qualidade de vida, sendo facilitadores de consequências psicossociais impostos por essa condição. Num estudo realizado por Reis RK, et al. (2017) cujo objetivo era analisar os sintomas somáticos e afetivo-cognitivos de depressão segundo o sexo de pessoas que vivem com HIVIAIDS, os resultados apontaram que as mulheres apresentam maiores escores de sintomatologia depressiva do que os homens, tanto no domínio somático quanto no afetivo/cognitivo das subescalas do Inventário de Depressão de Beck.

Numa pesquisa realizada por Beltrão RPL, et al. (2020) cujo objetivo era analisar a qualidade de vida e as dificuldades enfrentadas pelas pessoas que vivem com o vírus da Imunodeficiência Humana/Síndrome da Imunodeficiência Adquirida (HIVIAIDS) (PVHA). Foi apontado que o transtorno psiquiátrico, é condição frequente relacionado a vivência do HIVIAIDS, sendo a depressão 2 vezes mais prevalente atingindo entre 11 e $30 \%$ deste segmento quando comparado a população geral.

Alves APB e Pellegrini MA (2019) delinearam um estudo com objetivo de conhecer os processos de autoatenção e os fatores que influenciam nos itinerários terapêuticos no contexto de pessoas soropositivas, e compreender as relações sociais e os significados na experiência de saúde/doença. Utilizando a pesquisa qualitativa com base nas narrativas dos participantes, constatou-se através destas que a depressão é o transtorno psiquiátrico mais presente em suas vivências. 
Dias WB, et al. (2020) realizaram um estudo com intuito de descrever a vivência de acadêmicos durante uma atuação em um campo psicossocial, expondo observações e intervenções realizadas junto a usuários com HIVIAIDS. Constatando que transtornos psíquicos clássicos, como os de humor, principalmente depressão e ansiedade, estão estritamente associadas a pessoas que vivem com HIVIAIDS. Existindo uma prevalência desse transtorno na população infectada pelo vírus maior do que na população geral.

Num estudo realizado por Mello CJFA, et al. (2020) como objetivo identificar causas de abandono a Terapia Antirretroviral (TARV) em pessoas vivendo com HIVIAIDS no estado do Amapá. Os resultados apontaram os transtornos psiquiátricos (18\%) como fator de abandono ao tratamento. Sabe-se mundialmente que pessoas vivendo com HIVIAIDS são duas vezes mais acometidos pela depressão que a população geral. Pois esta pode ocorrer em diversas fases da infecção impactando na capacidade de abandono ao tratamento, qualidade de vida, progressão e agravamento da doença e consequente aumento da mortalidade. O que diverge do estudo de Silva MBG, et al. (2020) que apontou que os participantes que tinham carga viral indetectável, apresentavam melhor Qualidade de Vida em relação ao domínio Psicológico.

Um estudo realizado por Lemons A, et al. (2018) com objetivo de apresentar a primeira estimativa nacional dos aspectos sociodemográficos, clínicos e características comportamentais de homens transexuais HIV positivos recebendo cuidados médicos nos Estados Unidos. Constatou-se que $23 \%$ preenchiam os critérios para depressão. Pokhrel KN et al. (2019) delinearam um estudo para mensurar os sintomas depressivos, ansiedade, níveis de estresse, uso de drogas e não adesão a Terapia antirretroviral (TARV) entre pessoas vivendo com HIV no Nepal. Verificou-se que experimentar sintomas depressivos foi associado positivamente à não adesão ao TARV entre pessoas HIV-positivas.

Mandell LN, et al. (2019) conduziram uma pesquisa com o intuito de examinar os fatores de risco para ideação suicida entre adultos infectados pelo HIV em acompanhamento na Argentina. Os resultados apontam que $21 \%$ dos participantes relataram ideação suicida na semana anterior, o aumento da sintomatologia depressiva e o abuso de drogas foram associados significativamente a ideação suicida.

Bassett SM, et al. (2019) apresentaram dados piloto sobre a aceitabilidade e viabilidade de uma intervenção online para aumentar afeto daqueles que vivem com o vírus da imunodeficiência humana (HIV) em comorbidade com a depressão. Os resultados apontaram que o afeto positivo aumentou singnificativamente no grupo de intervenção.

Luo S, et al. (2020) propuseram um estudo que visava investigar mediação de atitudes negativas em relação ao envelhecimento em idosos com HIV e depressão na China continental. Os resultados apontaram a prevalência de depressão provável (CES-D $\geq 16$ ) foi significativamente maior no grupo HIV positivo do que no grupo HIV negativo (44,8\% versus $20,4 \%)$. Os participantes HIV-positivos apresentaram atitudes mais negativas em relação ao envelhecimento (em perda psicossocial e mudança física) do que seus colegas HIVnegativos. Atitudes negativas em relação ao envelhecimento foram associadas a mais sintomas depressivos. Atitudes gerais em relação ao envelhecimento (tamanho do efeito $=41,3 \%$ ) e os subdomínios de perda psicossocial (tamanho do efeito $=38,5 \%$ ) e mudança física (tamanho do efeito $=16,3 \%$ ) mediaram parcialmente a associação entre o status do HIV e sintomas depressivos, respectivamente.

Kagee A, et al. (2020) avaliaram o transtorno depressivo maior em pacientes recebendo cuidados de HIV em duas unidades de saúde pública na África do Sul usando a escala de depressão revisada do Centro de Estudos Epidemiológicos (CESD-R) e a Entrevista Clínica Estruturada para o DSM5. Dos 668 participantes do estudo, 229 (33,3\%; IC= 29,8\%; 36,9\%) pontuaram na faixa elevada no CESD-R e 170 (24,7\%; IC= 21,5\% a $28,1 \%$ ) preencheram os critérios diagnósticos para a depressão maior.

Zhu M, et al. (2020) concluíram um estudo que teve como objetivo examinar a associação entre o estigma relacionado ao HIV e a Qualidade de Vida (QV) e os efeitos indiretos do enfrentamento positivo e percepção de estresse em pessoas que vivem com HIV e sintomas depressivos. As análises mostraram que o estigma percebido e internalizado teve efeito negativo direto $(\beta=-0,14, p<0,05)$ na $Q V$. Além disso, o estigma percebido e internalizado teve efeitos indiretos na QV através da diminuição do enfrentamento positivo e aumento do estresse perecebido $(\beta=-0,23, p<0,001)$. 
Num estudo realizado por Nogueira GS e Seidl EMF (2016), que tinha como objetivo investigar a correlação entre percepção de doença e ansiedade, depressão e autoeficácia em pessoas vivendo HIV/AIDS. Os resultados apontaram uma correlação positiva e estatisticamente significativa entre percepção de doença e ansiedade $(r=0,64 ; p<0,01)$ e depressão $(r=0,68 ; p<0,01)$. Também foi constatado correlação negativa estatisticamente significativa entre percepção de doença e autoeficácia ( $r s=-0,41 ; p<0,05)$, indicando que quanto menor a autoeficácia percebida para aderir aos medicamentos antirretrovirais, maior a percepção de ameaça determinada pela doença.

Leite MAP, et al. (2020) delinearam um estudo cujo objetivo era investigar a esperança de vida e a depressão em pessoas vivendo com HIV/AIDS. Os resultados demonstram quanto ao tipo de exposição que culminou no diagnóstico de HIV/AIDS $88,2 \%$ apontaram para o sexo sem proteção, quanto ao estado civil a maioria foi de solteiros $58,8 \%$ (10). Os índices relacionados a esperança de vida indicaram escore médio $38,47 \pm 7,45$. Concluindo que a fé e a esperança possuem importantes valores, colaborando na capacidade de enfrentar com situações complicadas e na preservação da qualidade de vida. Portanto, facilitadoras dos impactos proporcionados por essa condição.

Arruda ACS e Coutinho DJG (2021) realizaram uma revisão integrativa da literatura cuja proposta era avaliar o risco para o desenvolvimento da depressão em pessoas vivendo com HIV/AIDS. Os resultados apontaram que a AIDS se configura como uma doença crônica na qual seus portadores possuem um risco alto para desenvolver sintomas depressivos. Sendo ocasionado principalmente pelo estigma e preconceito advindo da sociedade.

Patricio ACFA, et al. (2019) propuseram um estudo com objetivo de analisar depressão, auto-estima, expectativa futura e esperança de vida de pessoas com HIV/AIDS. Os resultados indicaram $31,5 \%$ de depressão leve, $21,3 \%$ moderada; $63 \%$ dos sujeitos mencionaram dificuldade de emprego digno; $58,8 \%$ manifestaram ser a vida um fracasso; $52,8 \%$ percebem-se inúteis. Medo, culpa e solidão contribuem para autoestima $(p \leq 0,05)$. A solidão contribui para esperança de vida $(p \leq 0,05)$.

No que se refere a depressão em idosos vivendo com HIVIAIDS Catalan J, et al. (2017) constataram numa amostra de 100 entrevistados que $12 \%$ possuíam sintomas de depressão e ansiedade e pacientes que não tem netos usufruem de melhor qualidade de vida. Porém numa pesquisa realizada com 335 homens idosos LGBT, quase $60 \%$ já haviam recebido diagnóstico de depressão e constatou-se que depressão e resiliência não possuem associação (EMLET CA, et al., 2017).

\section{CONSIDERAÇÕES FINAIS}

O número de pessoas vivendo com HIVIAIDS vem crescendo e esse crescimento vem acompanhado de alta prevalência de sinais e sintomas depressivos. Apoio social, familiar e trabalho são fatores de proteção para a qualidade de vida e saúde mental dos afetados. Manter o interesse de pesquisadores sobre a temática é indispensável frente aos quase 170.000 casos de AIDS registrados no Brasil pelo Boletim Epidemiológico do Ministério da Saúde no ano 2020. A luta contra a AIDS e os transtornos mentais decorrentes exige novos esforços e políticas governamentais, resiliência, persistência e incentivo à comunidade acadêmica, bem como o engajamento da sociedade civil. Além disso, é importante o apoio e participação ativa dos profissionais da área da saúde com vistas a diminuir o sofrimento daqueles que convivem com o HIVIAIDS.

\section{REFERÊNCIAS}

1. ALVES APB, PELLEGRINI MA. Convivendo com a infecção pelo vírus da imunodeficiência humana em Boa Vista (RR): um estudo sobre autoatenção e itinerários terapêuticos. Revista Eletrônica Acervo Saúde, 2019; 11(13): e941.

2. APA. American Psychiatric Association. Manual diagnóstico e estatístico de transtornos mentais $-D S M-5$. $5^{\mathrm{a}}$ Ed. Porto Alegre. Artmed, 2014; 992p.

3. ARRUDA ACS, COUTINHO DJG. Risco para depressão entre pacientes convivendo com HIV-AIDS. Revista Eletrônica Acervo Saúde, 2021; 13(4): e6908.

4. BALDIN L, et al. Sujeitos vivendo com a infecção por HIVIAIDS: um estudo qualitativo à luz da psicanálise. In Atas do Congresso Ibero-Americano de Investigação Qualitativa, Investigação Qualitativa em Saúde - CIAIQ 2, 2016. 
5. BASSETT SM, et al. Feasibility and acceptability of an online positive affect intervention for those living with comorbid HIV depression. AIDS and Behavior, 2019; 23(3): 753-764.

6. BELTRÃO RPL, et al. Saúde e qualidade de vida das pessoas vivendo com HIVIAIDS: uma revisão narrativa dos últimos 15 anos. Revista Eletrônica Acervo Saúde, 2020; 40: e2942-e2942.

7. BRASIL. Pesquisa Nacional por Amostra de Domicílios. Instituto Brasileiro de Geografia e Estatística - IBGE. 2010. Disponível em https://www.who.int/intellectualproperty/events/HIVAIDSPedroChequer.pdf. Acessado em: 6 de maio de 2021.

8. BRASIL. Programa Nacional de DST-AIDS do Ministério da Saúde. 2020. Disponível em https://bvsms.saude.gov.br/bvs/publicacoes/cd03 17.pdf. Acessado em 6 de maio de 2021.

9. BRIAN KB, et al. Suicidal ideation, suicide attempts, and HIV Infection. Psychosomatics. 1998; 39(5): 405-415.

10. CAMARGO LA, et al. Saúde mental, suporte familiar e adesão ao tratamento: associações no contexto HIVIAids. Psico-USF, 2014; 19(2): 221-232.

11. CAMPIOTTO LG, et al. Síndrome da imunodeficiência adquirida em idosos brasileiros. Revista Uningá Review, 2013; 16(1): 34-38.

12. CATALAN J, et al. What influences quality of life in older people living whit HIV? AIDS Research and Therapy, 2017; Canadá, 14(1): 1-10.

13. COUTINHO MFC et al. Tratamento antiretroviral: adesão e a influência da depressão em usuários com HIVIAIDS atendidos na atenção primária. Saúde em Debate [on line], 2018; 42(116): 148-161.

14. DIAS WB, et al. O perfil psicossocial de pessoas vivendo com HIVIAIDS em uma unidade de acompanhamento em Belém-PA: relato de experiência. Revista Eletrônica Acervo Saúde, 2020; 12(1): e1429.

15. EMLET CA, et al. Bouncing back: resilience and mastery among HIV positive older gav and bisexual men. The Gerontologist, 2017; 57(S1): 40-49.

16. JARDIM E. A doença e o tempo: AIDS, uma história de todos nós. Bazar do Tempo, 2019.

17. KAGEE A, et al. Predicting caseness of major depressive disorder using the Center for Epidemiological Studies Depression Scale (CESD-R) among patients receiving HIV care. General Hospital Psychiatry. 2020; 67: 70-76.

18. KAPLAN HI, SADOCK B. Compêndio de psiquiatria. 11th Ed. Porto Alegre. Artmed, 2016.

19. LEITE MAP, et al. Esperança de vida e depressão: pessoas vivendo com HIVIAids. Rev. Pesqui. (Univ. Fed. Estado Rio J., Online), 2020; 123-128.

20. LEMONS A, et al. Characteristics of HIV-positive transgender men receiving medical care: United States, 20092014. American Journal of Public Health, 2018; 108(1): 128-130.

21. LUO S, et al. Negative attitudes toward aging mediated the association between HIV status and depression among older people in mainland china. Journal of Affective Disorders, 2020; 277: 1005-1012.

22. MANDELL LN, et al. Suicidal ideation among adults re-engaging in HIV care in Argentina. AIDS and Behavior, 2019; 23(12): 3427-3434.

23. MELLO CJFA, et al. Terapia antirretroviral: principais causas de abandono no estado do Amapá. Revista Eletrônica Acervo Saúde, 2020; 12(8): e3423.

24. NOGUEIRA GS, SEIDL EMF. Associação entre percepção de doença e ansiedade, depressão e autoeficácia em pessoas com HIV/Aids. Temas em Psicologia, 2016; 24(2) 595-608.

25. OLIVEIRA LS, et al. A depressão como uma realidade dos dias atuais. In Anais da XIII FAVE, Fórum Acadêmico da Faculdade Vertice-Univetix, Matipó-MG, 2020.

26. OMS. Organização Mundial de Saúde. Classificação de transtornos mentais e de comportamento: CID-10. Porto Alegre. Artmed, 1993

27. OLIVEIRA LS, et al. A depressão como uma realidade dos dias atuais. In Anais da XIII FAVE, Fórum Acadêmico da Faculdade Vertice-Univetix, Matipó-MG, 2020.

28. OMS. Organização Mundial de Saúde. Depressão: o que você precisa saber. Disponível em https://www.paho.org/bra/index.php?option=com...depressao..Acesso em: 22 Dez. 2020.

29. PATRICIO ACFA, et al. Depressão, autoestima, expectativa futura e esperança de vida de pessoas com HIV. Revista Brasileira de Enfermagem, 2019; 72(5): 1354-60

30. PEDROSA SC, et al. Suporte social de pessoas que vivem com a síndrome da imunodeficiência adquirida. Texto \& Contexto Enfermagem, 2016; 25(4), 1-8

31. POKHREL KN et al. Mental health disorders and substance use among people living with HIV in Nepal: their influence on non-adherence to anti-retroviral therapy. AIDS Care, 2019; 31(8): 923-931.

32. REIS RK, et al. Sintomas de depressão e qualidade de vida de pessoas vivendo com HIV/aids. Revista LatinoAmericana de Enfermagem [Internet], 2011; 19(4): 874-881.

33. REIS RK, et al. Avaliação dos sintomas depressivos somáticos e afetivo-cognitivos de pessoas vivendo com HIVIAIDS. Acta Paulista de Enfermagem, 2017; 30(1), 60-65.

34. SILVA IBN, et al. Hope for life and depression: people living with HIVIAids. Revista de Pesquisa: Cuidado e Fundamental, 2020; 12(1): 124-129.

35. SILVA MBG, et al. Qualidade de vida dos portadores de HIVIAIDS no extremo norte do Brasil. Revista Eletrônica Acervo Saúde, 2020; 53(Sup.): e3757.

36. ROCHA RFC. Depressão entre acadêmicos do curso de enfermagem segundo o inventário de Beck. São Luiz, 2017. Disponível em: http://hdl.handle.net/123456789/2021. Acesso em: 23 Dez. 2020.

37. UNAIDS. United Nations Programme on HIVIAids. AIDS Epidemic Update, 2020. Disponível em

38. WHITBOURNE SK, HALGIN RP. Psicopatologia: perspectivas clínicas dos transtornos psicológicos. 7th Ed. Porto Alegre. AMGH, 2015: 162-183.

39. WORLD HEALTH ORGANIZATION (WHO). Depression and other common mental disorders: Global Health Estimates, 2017.

40. ZHU M, et al. HIV-related stigma, and quality of life in people living with HIV and depressive symptoms: indirect effects of positive coping and perceived stress. AIDS Care, 2020; 32(8): 1030-1035. 